\title{
10 devastating consequences of psychotic relapses
}

It breaks my heart every time young patients with functional disability and a history of several psychotic episodes are referred to me. It makes me wonder why they weren't protected from a lifetime of disability with the use of one of the FDA-approved longacting injectable (LAI) antipsychotics right after discharge from their initial hospitalization for first-episode psychosis (FEP).

Two decades ago, psychiatric research discovered that psychotic episodes are neurotoxic and neurodegenerative, with grave consequences for the brain if they recur. Although many clinicians are aware of the high rate of nonadherence in patients with schizophrenia-which inevitably leads to a psychotic relapse-the vast majority $(>99 \%$, in my estimate) never prescribe an LAI after the FEP to guarantee full adherence and protect the patient's brain from further atrophy due to relapses. The overall rate of LAI antipsychotic use is astonishingly low (approximately 10\%), despite the neurologic malignancy of psychotic episodes. Further, LAIs are most often used after a patient has experienced multiple psychotic episodes, at which point the patient has already lost a significant amount of brain tissue and has already descended into a life of permanent disability.
Oral antipsychotics have the same efficacy as their LAI counterparts, and certainly should be used initially in the hospital during FEP to ascertain the absence of an allergic reaction after initial exposure, and to establish tolerability. Inpatient nurses are experts at making sure a reluctant patient actually swallows the pills and does not cheek them to spit them out later. So patients who have had FEP do improve with oral medications in the hospital, but all bets are off that those patients will regularly ingest tablets every day after discharge. Studies show patients have a high rate of nonadherence within days or weeks after leaving the hospital for FEP. ${ }^{1}$ This leads to repetitive psychotic relapses and rehospitalizations, with dire consequences for young patients with schizophrenia-a very serious brain disorder that had been labeled "the worst disease of mankind" 2 in the era before studies showed LAI secondgeneration antipsychotics for FEP had remarkable rates of relapse prevention and recovery.,

Psychiatrists should approach FEP the same way oncologists approach cancer when it is diagnosed as Stage 1. Oncologists immediately take action to prevent the recurrence of the patient's cancer with chemotherapy and/or radiation therapy, and do not wait for the cancer to advance to Stage 4 , with widespread metastasis, before administering these potentially life-saving therapies (despite their toxic adverse effects). In

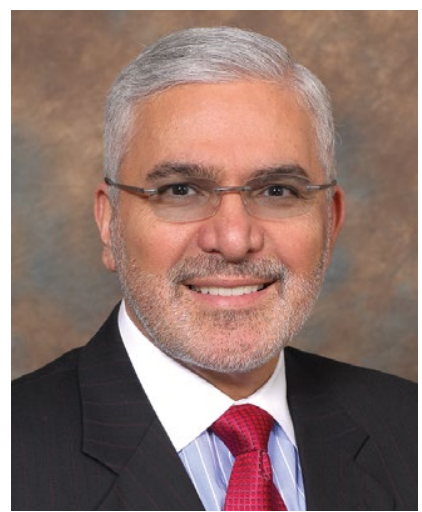

Henry A. Nasrallah, MD Editor-in-Chief doi: 10.12788/cp.0122

Starting a long-acting injectable antipsychotic immediately after firstepisode psychosis may prevent a lifetime of functional disability 


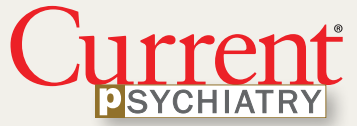

Editorial Staff

EDITOR Jeff Bauer

SENIOR EDITOR Sathya Achia Abraham

ASSISTANT EDITOR Jason Orszt

WEB EDITOR Kathryn Wighton

Art \& Production Staff

CREATIVE DIRECTOR Louise Koenig

ART DIRECTOR Pat Fopma

DIRECTOR, JOURNAL MANUFACTURING Michael Wendt

production MANAGER Donna Pituras

Publishing Staff

PUBLISHER Sharon Finch

DIRECTOR EBUSINESS DEVELOPMENT

Alison Paton

SENIOR DIRECTOR OF SALES

Tim LaPella

Editor-in-Chief Emeritus

James Randolph Hillard, MD

Frontline Medical Communications

VP, SALES Mike Guire

VP, DIGITAL CONTENT \& STRATEGY

Amy Pfeiffer

PRESIDENT, CUSTOM SOLUTIONS JoAnn Wahl

CIRCULATION DIRECTOR Jared Sonners

In affiliation with Global Academy for Medical Education, LLC

PRESIDENT David J. Small, MBA

\section{FRONTLNE MCedge}

7 Century Drive, Suite 302

Parsippany, NJ 07054

Tel: (973) 206-3434

Fax: (973) 206-9378

www.frontlinemedcom.com

Subscription Inquiries:

subscriptions@mdedge.com

Published through an

educational partnership with

léCINCINNATI

May 2021 schizophrenia, functional disability is the equivalent of Stage 4 cancer and should be aggressively prevented by using LAIs at the time of initial diagnosis, which is Stage 1 schizophrenia. Knowing the grave consequences of psychotic relapses, there is no logical reason whatsoever not to switch patients who have had FEP to an LAI before they are discharged from the hospital. A well-known study by a UCLA research group that compared patients who had FEP and were assigned to oral vs LAI antipsychotics at the time of discharge reported a stunning difference at the end of 1 year: a $650 \%$ higher relapse rate among the oral medication group compared with the LAI group! ${ }^{5}$ In light of such a massive difference, wouldn't psychiatrists want to treat their sons or daughters with an LAI antipsychotic right after FEP? I certainly would, and I have always believed in treating every patient like a family member.

\section{Catastrophic consequences}

This lack of early intervention with LAI antipsychotics following FEP is the main reason schizophrenia is associated with poor clinical and functional outcomes. Patients are prescribed pills that they often take erratically or not at all, and end up relapsing repeatedly, with multiple catastrophic consequences, such as:

1. Brain tissue loss. Until recently, psychiatry did not know that psychosis destroys gray and white matter in the brain and causes progressive brain atrophy with every psychotic relapse. ${ }^{6,7}$ The neurotoxicity of psychosis is attributed to 2 destructive processes: neuroinflammation ${ }^{8,9}$ and free radicals. ${ }^{10}$ Approximately $11 \mathrm{cc}$ of brain tissue is lost during FEP and with every subsequent relapse. ${ }^{6}$ Simple math shows that after 3 to 5 relapses, patients' brains will shrink by $35 \mathrm{cc}$ to $60 \mathrm{cc}$. No wonder recurrent psychoses lead to a life of permanent disability. As I have said in a past editorial, ${ }^{11}$ just as cardiologists do everything they can to prevent a second myocardial infarction ("heart attack"), psychiatrists must do the same to prevent a second psychotic episode ("brain attack").

2. Treatment resistance. With each psychotic episode, the low antipsychotic dose that worked well in FEP is no longer enough and must be increased. The neurodegenerative effects of psychosis implies that the brain structure changes with each episode. Higher and higher doses become necessary with every psychotic recurrence, and studies show that approximately 1 in 8 patients may stop responding altogether after a psychotic relapse. ${ }^{12}$

3. Disability. Functional disability, both vocational and social, usually begins after the second psychotic episode, which is why it is so important to prevent the second episode. ${ }^{13}$ Patients usually must drop out of high school or college or quit the job they held before FEP. Most patients with multiple psychotic episodes will never be able to work, get married, have children, live independently, or develop a circle of friends. Disability in schizophrenia is essentially a functional death sentence. ${ }^{14}$

4. Incarceration and criminalization. So many of our patients with schizophrenia get arrested when they become psychotic and behave erratically due to delusions, hallucinations, or both. They typically are taken to jail instead of a hospital because almost all the state hospitals around the country have been closed. It is outrageous that a medical condition of the brain leads to criminalization of patients with schizophrenia. ${ }^{15}$ The only solution for this ongoing crisis of incarceration of our patients with schizophrenia is to prevent them from relapsing into psychosis. The so-called deinstitutionalization movement has mutated into trans-institutionalization, moving patients who are medically ill from state 
hospitals to more restrictive state prisons. Patients with schizophrenia should be surrounded by a mental health team, not by armed prison guards. The rate of recidivism among these individuals is extremely high because patients who are released often stop taking their medications and get re-arrested when their behavior deteriorates.

5. Suicide. The rate of suicide in the first year after FEP is astronomical. A recent study reported an unimaginably high suicide rate: $17,000 \%$ higher than that of the general population. ${ }^{16}$ Many patients with FEP commit suicide after they stop taking their antipsychotic medication, and often no antipsychotic medication is detected in their postmortem blood samples.

6. Homelessness. A disproportionate number of patients with schizophrenia become homeless. ${ }^{17}$ It started in the 1980s, when the shuttering of state hospitals began and patients with chronic illnesses were released into the community to fend for themselves. Many perished. Others became homeless, living on the streets of urban areas.

7. Early mortality. Schizophrenia has repeatedly been shown to be associated with early mortality, with a loss of approximately 25 potential years of life. ${ }^{17}$ This is attributed to lifestyle risk factors (eg, sedentary living, poor diet) and multiple medical comorbidities (eg, obesity, diabetes, hypertension). To make things worse, patients with schizophrenia do not receive basic medical care to protect them from cardiovascular morbidity, an appalling disparity of care. ${ }^{18}$ Interestingly, a recent 7-year follow-up study of patients with schizophrenia found that the lowest rate of mortality from all causes was among patients receiving a second-generation LAI. ${ }^{19}$ Relapse prevention with LAIs can reduce mortality! According to that study, the worst mortality rate was observed in patients with schizophrenia who were not receiving any antipsychotic medication.

\section{Posttraumatic stress disorder (PTSD).} Many studies report that psychosis triggers PTSD symptoms ${ }^{20}$ because delusions and hallucinations can represent a life-threatening experience. The symptoms of PTSD get embedded within the positive and negative symptoms of schizophrenia, and every psychotic relapse serves as a "booster shot" for PTSD, leading to depression, anxiety, personality changes, aggressive behavior, and suicide.

9. Hopelessness, depression, and demoralization. The stigma of a severe psychiatric brain disorder such as schizophrenia, with multiple episodes, disability, incarceration, and homelessness, extends to the patients themselves, who become hopeless and demoralized by a chronic illness that marginalizes them into desperately ill individuals. ${ }^{21}$ The more psychotic episodes, the more intense the demoralization, hopelessness, and depression.

10. Family burden. The repercussions of psychotic relapses after FEP leads to significant financial and emotional stress on patients' families. ${ }^{22}$ The heavy burden of caregiving among family members can be highly distressing, leading to depression and medical illness due to compromised immune functions.

\section{Preventing relapse: It is not rocket science}

It is obvious that the single most important therapeutic action for patients with schizophrenia is to prevent psychotic relapses. Even partial nonadherence must be prevented, because a drop of $25 \%$ in a patient's serum antipsychotic level has been reported to lead to a psychotic relapse. ${ }^{23}$ Preventing relapse after FEP is not rocket science: Switch the patient to an LAI before discharge from

\section{There is no logical reason whatsoever not to switch a patient who has had FEP to a long-acting injectable antipsychotic before they are discharged from the hospital}


I have witnessed

firsthand how stable and

functional a patient who

has had FEP can become

when started on an LAI

very soon after the onset of the illness the hospital, ${ }^{24}$ and provide the clinically necessary psychosocial treatments at every monthly follow-up visit (supportive psychotherapy, social skill training, vocational rehabilitation, and cognitive remediation). I have witnessed firsthand how stable and functional a patient who has had FEP can become when started on a second-generation LAI very soon after the onset of the illness.

I will finish with a simple question to my clinician readers: given the many devastating consequences of psychotic relapses, what would you do for your young patient with FEP? I hope you will treat them like a family member, and protect them from brain atrophy, disability, incarceration, homelessness, and suicide by starting them on an LAI antipsychotic before they leave the hospital. We must do no less for this highly vulnerable, young patient population.

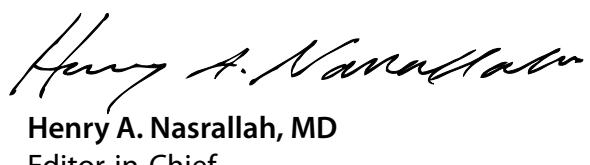

References

1. Velligan DI, Sajatovic M, Hatch A, et al. Why do psychiatric patients stop antipsychotic medication? A systematic review of reasons for nonadherence to medication in patients with serious mental illness. Patient Prefer Adherence. 2017;11:449-468.

2. Where next with psychiatric illness? Nature. 1988; 336(6195):95-96.

3. Emsley R, Oosthuizen P, Koen L, et al. Remission in patients with first-episode schizophrenia receiving assured antipsychotic medication: a study with risperidone long-acting injection. Int Clin Psychopharmacol. 2008;23(6):325-331

4. Kishimoto T, Hagi K, Kurokawa S, et al. Longacting injectable versus oral antipsychotics for the maintenance treatment of schizophrenia: a systematic review and comparative meta-analysis of randomised, cohort, and pre-post studies. Lance Psychiatry. 2021:S2215-0366(21)00039-0. doi: 10.1016/ S2215-0366(21)00039-0

5. Subotnik KL, Casaus LR, Ventura J, et al. Long-acting injectable risperidone for relapse prevention and control of breakthrough symptoms after a recent first episode of schizophrenia. A randomized clinical trial. JAMA Psychiatry. 2015;72(8):822-829.

6. Cahn W, Hulshoff Pol HE, Lems EB, et al. Brain volume changes in first-episode schizophrenia: a 1-year follow-up study. Arch Gen Psychiatry. 2002;59(11):1002-1010.

7. Lei W, Kirkpatrick B, Wang Q, et al. Progressive brain structural changes after the first year of treatment in first-episode treatment-naive patients with deficit or nondeficit schizophrenia. Psychiatry Res Neuroimaging. 2019;288:12-20

8. Monji A, Kato TA, Mizoguchi Y, et al. Neuroinflammation in schizophrenia especially focused on the role of microglia. Prog Neuropsychopharmacol Biol Psychiatry. 2013;42:115-121.

9. Köhler-Forsberg O, Müller N, Lennox BR. Editorial The role of inflammation in the etiology and treatment of schizophrenia. Front Psychiatry. 2020;11:603296. doi: 10.3389 /fpsyt.2020.603296

10. Noto C, Ota VK, Gadelha A, et al. Oxidative stress in drug naïve first episode psychosis and antioxidant effects of risperidone. J Psychiatr Res. 2015;68:210-216.

11. Nasrallah HA. For first-episode psychosis, psychiatrists should behave like cardiologists. Current Psychiatry. 2017;16(8):4-7.

12. Emsley R, Oosthuizen P, Koen L, et al. Comparison of treatment response in second-episode versus firstepisode schizophrenia. J Clin Psychopharmacol. 2013, 33(1):80-83

13. Alvarez-Jiménez M, Parker AG, Hetrick SE, et al. Preventing the second episode: a systematic review and meta-analysis of psychosocial and pharmacological trials in first-episode psychosis. Schizophr Bull. 2011;37(3):619-630.

14. Weye N, Santomauro DF, Agerbo E, et al. Registerbased metrics of years lived with disability associated with mental and substance use disorders: a registerbased cohort study in Denmark. Lancet Psychiatry. 2021;8(4):310-319.

15. Kirchebner J, Günther MP, Lau S. Identifying influential factors distinguishing recidivists among offender patients with a diagnosis of schizophrenia via machine learning algorithms. Forensic Sci Int 2020;315:110435. doi: 10.1016/j.forsciint.2020.110435

16. Zaheer J, Olfson M, Mallia E, et al. Predictors of suicide at time of diagnosis in schizophrenia spectrum disorder: a 20-year total population study in Ontario, Canada. Schizophr Res. 2020;222: 382-388.

17. Colton CW, Manderscheid RW. Congruencies in increased mortality rates, years of potential life lost, and causes of death among public mental health clients in eight states. Prev Chronic Dis. 2006;3(2):A42.

18. Nasrallah HA, Meyer JM, Goff DC, et al. Low rates of treatment for hypertension, dyslipidemia and diabetes in schizophrenia: data from the CATIE schizophrenia trial sample at baseline. Schizophr Res. 2006;86(1-3):15-22.

19. Taipale H, Mittendorfer-Rutz E, Alexanderson K, et al Antipsychotics and mortality in a nationwide cohort of 29,823 patients with schizophrenia. Schizophr Res. 2018;197:274-280.

20. Seedat S, Stein MB, Oosthuizen PP, et al. Linking posttraumatic stress disorder and psychosis: a look at epidemiology, phenomenology, and treatment. J Nerv Ment Dis. 2003;191(10):675-681.

21. Berardelli I, Sarubbi S, Rogante E, et al. The role of demoralization and hopelessness in suicide risk in schizophrenia: A review of the literature. Medicina (Kaunas). 2019;55(5):200.

22. Khalil SA, Elbatrawy AN, Saleh NM, et al The burden of care and burn out syndrome in caregivers of an Egyptian sample of schizophrenia patients. Int J Soc Psychiatry. 2021;10. doi: 10.1177/ 0020764021993155

23. Subotnik KL, Nuechterlein $\mathrm{KH}$, Ventura J, et al Risperidone nonadherence and return of positive symptoms in the early course of schizophrenia. Am J Psychiatry. 2011;168(3):286-292.

24. Garner KN, Nasrallah HA. Managing first-episode psychosis: Rationale and evidence for nonstandard first-line treatments for schizophrenia. Current Psychiatry. 2015;14(7):33-45. 